\title{
KÖRPERSPRACHE IM KONTEXT \\ DES KOMMUNIKATIONSPROZESSES \\ BODY LANGUAGE IN THE CONTEXT \\ OF THE COMMUNICATION PROCESS
}

Mihaela PARPALEA ${ }^{1}$

\begin{abstract}
This paper presents several research-based aspects on language-related communication, message-related communication and action-related communication and the connection between inner and outer attitude within communication, meaning that mental and physical conditions of speaking and hearing go hand in hand. The article also describes some differences between verbal and non-verbal communication, as well as encoding and decoding procedures in inter-cultural communication. Communication is about acting, with and without language, about intentions, about the circumstances and relationships between people, about attitudes behind the words, about physical behavior that expresses inner attitudes. Feelings are expressed in body language and physical changes also change the emotional state of communication participants. Summing up, some views of what communicative language and action are and are not, of what they can and cannot, are also presented.
\end{abstract}

Keywords: verbal and non-verbal communication, vocal and non-vocal communication, communicative behavior, linguistic action, gestures.

DOI: $10.24818 / \mathrm{SYN} / 2021 / 17 / 2.08$

\section{Einleitung}

$\mathrm{Zu}$ den Merkmalen unserer Zeit gehört ein gesteigertes Körperbewusstsein, das dazu führt, dass menschlicher Körper mehr als nur Natur bedeutet, weil er immer mehr von kulturellen Werten geprägt ist. Im Laufe der Geschichte hat das menschliche Körperverständnis Wandlungen durchgemacht, so war der Körper im Mittelalter als Gefängnis der Seele betrachtet, später standen in der abendländischen Kulturgeschichte Seele und Geist höher als der Körper und heute hat sich der Blick von der Seele immer mehr auf den Körper gerichtet. Weil der Mensch heutzutage seinem Aussehen große Bedeutung beimisst, so ist Körperkultur beinahe in Körperkult umgeschlagen. Nicht Körperbau, Aussehen und Schönheit, sondern die Ausdrucksmerkmale des Körpers, die auf den Menschen, auf seine Wesensart schließen lassen, Gestik und Mimik, Körpersprache, gestisches Sprechen, körperliches Verhalten im kommunikativen Handeln sind das Thema des vorliegenden Artikels.

\footnotetext{
${ }^{1}$ Mihaela Parpalea, Transilvania University of Brașov, mihaela.parpalea@unitbv.ro
} 


\section{Vielfältigkeit der Kommunikationsmöglichkeiten}

\subsection{Kommunikation im weiteren Sinn}

Was menschliche Kommunikation ist, wissen wir alle. Sie ist alltägliche Praxis, eine Bedingung für die Möglichkeit von Gesellschaft, Teil der Natur des Menschen. Das soziale Leben, die soziale Verständigung bedarf des Austausches von Gedanken und Ideen in der alltäglichen Praxis. Jede Gesellschaft verfügt über eine Vielzahl von Ausdrucksmöglichkeiten, wie z.B. die natürlichen Sprachen und die Schrift, über Gestik und Mimik, Proxemik, ikonische Systeme, Piktogramme, Film, Malerei und Musik sowie auch über verschiedene Kodes des Essens, des Benehmens, der Kleidung, über religiöse Rituale usw. (vgl. Hübler, 2007).

In dieser Vielzahl von Äußerungsformen kooperieren mehrere Elemente. Dazu gehören in erster Linie die akustischen Elemente im Umgang mit Sprache, d.h. die Lautfolge der gesprochenen Sprache, als ein wichtiger Teil des Kommunikationsprozesses. Dieser Prozess ist eingebettet in das MiteinanderHandeln der Kommunikationsteilnehmer und er umfasst mehr als nur Planung, Kodierung, Artikulation, Formulierung, Verarbeitung von Informationen und Konzeptualisierung. Diese Teilprozesse sind zwar notwendig zur Repräsentation der Sachverhalte, aber dazu trägt auch die Körpersprache bei, sodass eine Vielfalt von Elementen wie Weltwissen, Situationskenntnis und soziale Interaktion der Kommunikation zugeteilt wird (vgl. Dietrich, 2002: 125-186). Zu den Äußerungsformen gehören außer der Sprache auch die gestisch-mimische Gebärdensprache, die Gesichtsausdrücke, die Körperhaltung sowie auch proxemisch-kinetische Elemente, die in den verschiedenen Alltagssituationen innerhalb der Kommunikation auftreten. Es ist wichtig zu erkennen, in welchen kommunikativen Kontexten sie auftreten, welche Äußerungen und Gesten zusammenpassen und wie sie zu verstehen sind.

Es gibt ein komplexes Zusammenspiel von Worten und Körpersprache in der zwischenmenschlichen Kommunikation. Man unterscheidet dabei nach sprachlichen, normativen, situativen, emotional bedingten, kulturellen und sozialen Kontexten, wenn man verschiedene Kommunikationssituationen analysieren soll.

Wie der Kommunikationsvorgang abläuft, erklären Linguisten, Psychologen und Sprachphilosophen, sodass in der Fachliteratur gezeigt wird, dass der Kommunikationsprozess gleichzeitig immer als Ursache und als Wirkung aufgefasst werden kann. Wie die Kommunikationspartner sich in der jeweiligen Kommunikationssituation verhalten, hängt von ihrer Einschätzung von Ursache und Wirkung des Verhaltens ab (vgl. Stadler, 1978: 47-73). Die Reaktion eines Kommunikationspartners verändert die sprachliche und gestische Folgereaktion des anderen Partners. Dieses hat eine veränderte Einschätzung der Absicht des 
einen Partners durch den anderen zur Folge, was sich im geänderten sprachlichen und gestischen Verhalten aufweist. Dabei treten Sprachkode, Klangbild, Satzmuster, syntaktische und semantische Normierung, Gestik und Mimik mit ins Spiel (vgl. Stadler, 1978: 62). In diesem Sinne sind Untersuchungen zur Spracherwerbsituation relevant sowie auch Untersuchungen über das geschlechtstypische Kommunikationsverhalten verschiedener sozialer und kultureller Schichten, über die Beeinflussung oder Machtausübung durch kommunikative Akte (vgl. Navarro, 2010).

\subsection{Konzepte des gestischen Sprechens}

Menschen drücken immer etwas aus, auch wenn sie sich nicht sprachlich äußern. Die Zeichen und die Signale, die ihr Körper vermitteln, lassen sich nicht abstellen. Diese drücken Haltungen aus und selbst die Umgangsformen, Kleidungen und Rituale sind Ausdrucksformen der Menschen. Die Körpersignale sind einerseits natürlich, angeboren, andererseits erlernt, erworben. Sowohl die einen, als auch die anderen Signale können schwer gezielt gesteuert werden. Menschen benutzen die Körpersprache, die ihr elementares Ausdrucksmittel ist, doch schätzen sie nicht immer alle ihre Möglichkeiten richtig ein. Viele nonverbale Kommunikationsmittel, als Teil der menschlichen Kommunikation, die sich nicht nach Konventionen der Sprache richten, lassen sich von ihr nicht beeinflussen. Bis auf einige Ausnahmen beim Ausdruck von Gefühlen, wie Freude, Wut spielen angeborene Verhaltensweisen der Menschen innerhalb der Gemeinschaft eine untergeordnete Rolle. Man kann behaupten, dass der Mensch unbewusst Botschaften mit Körpersignalen übermittelt.

Viele Untersuchungen, die verschiedene Verhaltensweisen innerhalb der Kommunikation betreffen, sind rein deskriptiv, andere hingegen sind kritisch angelegt und sollen eine Verbesserung der Kommunikation bewirken (vgl. Hübler, 2001: 26). Hier sind Informationen zum verbalen und nonverbalen Zeichensystem und zu Gesprächsanalysen im soziolinguistischen Kontext zu finden.

Zum Konzept des gestischen Sprechens erwähnt Hübler fünf Arten des nonverbalen Verhaltens. Die erste Art betrifft die Körperausdrücke, für die es eine sprachliche Entsprechung gibt wie z. B. den Ausdruck von Müdigkeit, Angst, Nervosität, wobei diese Ausdrucksmöglichkeiten kulturell unterschiedlich konventionalisiert sind. Diese Art des nonverbalen Verhaltens nennte Hübler „Embleme“. Die Studie zeigt, dass das nonverbale Verhalten mit dem verbalen übersetzbar ist. Die „Illustratoren“ sind die zweite Art des nonverbalen Verhaltens bei Hübler und er versteht darunter sprachbegleitende, unterstützende, ergänzende Ausdrucksbewegungen, die eigentlich die verbale Äußerung illustrieren mögen. Die dritte Art, die „Regulatoren“, steuern den Interaktionsfluss zwischen Sprecher und Hörer. Es sind körpersprachliche Ausdrücke mit Steuerungsfunktion. Die vierte Art betrifft die Gefühlsausdrücke, die die verbalen Ausdrücke unterstreichen, 
wiederholen oder widerlegen. Damit meint Hübler die Affektdarstellungen, die den Ausdruck von Emotionen ermöglichen können. Die fünfte Art des nonverbalen Verhaltens betrifft die sogenannten „Adaptoren“, die Verhaltensweisen sind, die den selbstbezogenen Bedürfnissen der Sprecher dienen. Es sind körpersprachliche Ausdrucksweisen, die Emotionen, denen der Sprecher nicht Herr wird, verbergen. Beispiele sind das Hantieren mit einem Füller während einer Präsentation vor einem Publikum, das Drehen am Jackenknopf oder am Ehering beim Vorlesen eines Textes. Diese fünf Arten des nonverbalen Verhaltens zeigen, dass die Körpersprache Auskunft über den Sprecher, über seine Befindlichkeit, über seine Beziehung zu den Hörern zeigt, dass Körpersprache mehr als nur ein Mittel zur Verbesserung einer Rede vor einem Publikum ist. Wissenschaftliche Untersuchungen haben nähere Aufschlüsse gebracht, genaues systematisches Wissen über die Kommunikation gewonnen, sodass dieses Wissen praktisch greifen kann, sodass wir besser und reflektierter kommunizieren, wenn wir dieses Wissen haben (vgl. Schmidt, 2019).

In der Linguistik und in der Sprachphilosophie wird der Begriff „Kommunikation“ in einer engeren, genaueren Bedeutung verwendet als im Alltag und bezeichnet die Art der Verständigung zwischen den Menschen. Keller definiert Kommunikation wie folgt:

Kommunikation ist jedes intentionale Verhalten, das in der Absicht vollzogen wird, dem anderen auf offene Weise etwas zu erkennen zu geben. Kommunizieren heißt Mitmenschen beeinflussen, und zwar dadurch, dass man dem anderen mittels Zeichen (im weitesten Sinne) zu erkennen gibt, wozu man ihn bringen möchte, in der Hoffnung, dass diese Erkenntnis für den anderen ein Grund sein möge, sich in der gewünschten Weise beeinflussen zu lassen. (Keller, 1994: 104)

Allerdings stellen sich hier noch Fragen, wie etwa, was ist ,intentionales Verhalten“, was heißt „offene Weise“, was sind „Zeichen im weitesten Sinne“? Menschen kommunizieren, die Gehirnzellen auch. Kommunikativ ist z.B. auch das Tragen einer Krawatte, wenn in einem bestimmten Kontext über einen Krawattenmuffel die Rede sein sollte. Kommunikation ist möglich über Zeichen verschiedener Art, über lautliche, graphische, gestische Zeichen. Ein Zeichen ist immer dann gegeben, wenn etwas über sich hinaus verweist, wenn ein Sachverhalt nicht nur diesen Sachverhalt bedeutet, sondern damit etwas anderes, darüber Hinausgehendes verbunden ist. Ein Beispiel dafür ist die Handhaltung, die Handfläche nach oben, der mittlere Finger gestreckt. Das ist Körpersprache, die übersetzt als sogenannter "Stinkefinger" bekannt ist. Sie hat eine bestimmte Bedeutung, die auch kulturell markiert ist. So heißt z.B. ein Kreis mit Zeigefinger und Daumen ein Lob in Mitteleuropa und eine Beleidigung in Südeuropa, einen Finger am Kopf halten, heißt in Nordamerika ein Kompliment, ganz anders ist das in den deutschsprachigen Ländern, wo es heißt „einen Vogel haben“, verrückt sein.

SYNERGY volume 17 , no. $2 / 2021$ 
Die Kommunikationsunterschiede zwischen Menschen und Kulturen sind oft sehr groß, obwohl Menschen fast die gleichen Verstehensfähigkeiten haben. Sie müssen nur richtig zuhören, schauen, einschätzen und offen sein, ja sogar mit Händen und Füßen reden, wenn nötig, um zu kommunizieren und sich verständlich zu machen.

\section{Kommunikatives Verhalten}

\subsection{Unterschiedliche Ausdrucksformen}

In der Fachliteratur werden vier Modalitäten kommunikativen Verhaltens beschrieben (vgl. Nörth, 1985: 322). Nörth unterscheidet zwischen vier Arten kommunikativen Verhaltens, zwischen verbal und nonverbal, vokal und nonvokal, aus deren Kombination Folgendes resultiert: verbale und vokale Kommunikation ist die Lautsprache, nonverbal und vokal ist der Bereich der Paralinguistik, verbal und nonvokal ist die Schrift und die Zeichensprache und nonverbal und nonvokal umfasst die Kommunikationsart, die visuelle, taktile Aspekte unterstreicht. Hierher gehören auch Körperhaltung und Körperposition, Bewegungen, wie Menschen gehen, wie sie sitzen. Wenn jemand unruhig und oft die Beine übereinanderschlägt oder mit dem Oberkörper sich ständig wiegt, so kann das Nervosität bedeuten. Sieht man Körperhaltung als Ausdruck von der Wahrnehmungsseite her, dann kann ein kommunikativer Akt vorliegen, auch wenn keine bestimmte Intentionalität dahintersteckt.

Nonverbale Verhaltenselemente und ihre Bezugnahme auf Sprache standen und stehen im Mittelpunkt des Forschungsinteresses, wo es heißt, dass der verbal und nonverbal kommunizierende Mensch als „Multikanal System“ (Helfrich und Wallbott, 1980: 267) bezeichnet werden kann. Die beiden Autoren zeigen, dass nonverbale Kommunikation nicht gleichzusetzen mit dem nonverbalen Verhalten ist. Die nonverbale Kommunikation vollzieht sich mittels eines Kodes, ähnlich dem der Sprache. Demnach gliedert sich die nonverbale Kommunikation in vokal und nonvokal genauso wie auch Nörth meinte. Vokal sind die Sprache, die Sprechdauer und die Stimmqualität, nonvokal sind Mimik, Gestik, Blick, Körperbewegung, Körperhaltung, Geschmack, Geruchsinn, Tastsinn Wärmeempfindung, interpersonale Distanz, Sitzverteilung, Kleidung, persönliche Aufmachung, Haare, Tattoo, Make-up usw. (vgl. Nörth, 1985: 325). Diese sagen einem etwas über eine Person. Die nonvokale Kommunikation unterstützt nur die vokale Kommunikation, ersetzt sie jedoch nicht. Wenn das stimmliche, das gestische und das mimische Verhalten innerhalb einer konversationalen Interaktion eingesetzt wird, dann hat es denselben Kodecharakter wie die Sprache. Der Kodecharakter der nonverbalen Kommunikation ist im Gegensatz zum sprachlichen Kode ontogenetisch und phylogenetisch viel fester markiert, denn es gibt viele unkonventionelle Aspekte, die der nonverbalen Kommunikation eigen sind. So kann man z.B. bei einem erfahrenen Sprecher verstehen, was für Gefühle er ausdrückt, selbst wenn man die 
Sprache, die er spricht, nicht kennt. Aus dem Tonfall, aus der Mimik und Gestik ist das Verstehen des Gesagten dann erkennbar. Ein Gefühl, eine Emotion kann enkodiert werden. Paul Watzlawick zeigt in seinen fünf Axiomen, dass Kommunikation entweder digital oder analog sein kann (vgl. Watzlawick, 1974). Nonverbale Kommunikation ist analog und hat einen allgemeinen Geltungsbereich. So bedeutet z.B. das Lächeln, dass jemand freundlich oder falsch, verlegen oder verlogen, triumphierend, wissend oder höflich, amüsiert oder ironisch ist, aber in verschiedenen Kulturen wird die Bedeutung des Lächelns unterschiedlich gewertet. Von der nonverbalen Kommunikation wird verlangt, dass sie den Prozess des Sendens nonverbaler Signale, deren Erscheinungsformen und Empfangen sowie auch die Verarbeitung dieser Signale zeigt.

\subsection{Nonverbale und vokale Kommunikation}

An seinem Arbeitsplatz und in der Freizeit hat der Mensch im Alltag bestimmte Rollen zu erfüllen. Das drückt sich auch in der Körpersprache aus. Jeder hat andere Verhaltensregeln zu befolgen. Für eine Sekretärin gelten andere Verhaltensregeln als für einen Arzt oder für einen Bankangestellten. In der jeweiligen Umgebung versprechen bestimmte Verhaltensmuster ein gewünschtes Handeln. Die gesellschaftlichen Normen zeigen sich in der Körpersprache und am Verhalten einer Person kann man oft ihre soziale Stellung, ihre Selbsteinschätzung, ihre Hochmütigkeit, ihre Menschenfreundlichkeit erkennen. Es sind konventionalisierte Verhaltensmuster, die in jedem Menschen stecken, ob sie wollen oder nicht. Diese Verhaltensmuster sind von der gesellschaftlichen Rolle der Person bedingt. So kann der Direktor die Sekretärin anschreien und dabei gestikulieren, so auch ein Bankangestellter den Portier, wenn diese ihre Pflicht nicht gewissenhaft tun. Der Schreiton ist das Untersuchungsobjekt der Paralinguistik. Die Körpersprache ist jedoch viel komplexer als es nur die Gesten oder der Schreiton erkennen lassen.

\subsection{Nonverbale und nonvokale Kommunikation}

Bei verschiedenen Veranstaltungen, Ritualien, Zeremonien sowie bei der Werbung werden körperliche Ausdrucksformen bewusst und geplant eingesetzt, weil die Gesten mehr und besser ausdrücken können, was mit Worten schwieriger erreichbar wäre.

Körperhaltung, Körperbewegungen finden Anwendung als Unterstützung der gesprochenen Sprache oder als Einsatz dafür. Durch die Körperhaltung werden auch Gefühle ausgedrückt, Einstellungen gegenüber Personen und Situationen.

Die nonverbale und nonvokale Kommunikation ist mindestens genauso wichtig wie die sprachliche und sprachlich-begleitende, wenn man den Zuhörer anspricht und einen bestimmten Eindruck erwecken möchte. Durch die Körpersprache drückt jeder unmittelbar seine Befindlichkeit aus. Das zeigt sich im Blickkontakt, in der 
interpersonalen Distanz, im Vorwärtsneigen des Körpers, Berühren, im Kreuzen der Arme, in der Entspanntheit der Hände, im Übereinanderschlagen der Beine. Auf die meisten dieser Körperhaltungen haben Menschen wenig Einfluss.

Der Blick ist eines der wichtigsten nonverbalen und wirksamsten Ausdrucksmitteln.

Ein langer Blick zeigt Interesse dem Kommunikationspartner gegenüber, ein zu langer und intensiver Blick signalisiert Aggression. Sicher spielt hier die Gesprächssituation eine wichtige Rolle, der Status und die Vertrautheit der Kommunikationspartner. Der Blickkontakt wird unterschiedlich eingeschätzt in den verschiedenen Kulturen, was ein wichtiger Aspekt der nonverbalen und nonvokalen Kommunikation ist.

Dasselbe kann auch über das Distanzverhalten gesagt werden. Wer, wem, wann und wie nahe stehen kann oder darf und erst recht wer wen berühren darf, unterliegt bestimmten Regeln. In vielen Kulturen spielt das eine sehr wichtige Rolle.

Körperbewegungen sind ebenfalls kulturabhängig. Kopf -und Handbewegungen sind gewöhnlich mit Betonung, Lautstärke Tonhöhe oder Kommentierung einzelner Worte und Argumenten verbunden.

\section{Nonverbale Kommunikation und nonverbales Verhalten}

Die nonverbale Kommunikation wird in verschiedenen kommunikationaswissenschaftlichen Disziplinen, in der Verhaltensforschung untersucht (vgl. Navarro, 2018). Sie umfasst die Gestik, die die Bewegungen der Hände, Arme und Finger sowie auch des Kopfes bedeutet. Es werden Art, Tempo, Zahl der Gesten gedeutet. Die nonverbale Kommunikation bezieht sich auch auf die Mimik. Es geht um die Bewegung der Gesichtsmuskeln als Ausdruck der Gemütsverfassung. Die Augen als der Spiegel der Seele, sagen oft mehr als man sprachlich formulieren mag. Ein Anblick kann freundlich, offen, interessiert, ironisch, frech, scharf sein. Er kann mehr ausdrücken als Worte es tun können. Er kann Zutrauen, Einverständnis, Misstrauen, Ärger ausdrücken.

Ein anderer Teil der nonverbalen Kommunikation ist die Körperhaltung und betrifft das Gesamtbild der Person, ist ein Ausdruck der Stimmung, ja sogar der Charaktermerkmale und der Hinwendung zum Gesprächspartner und zeigt, ob man in seinem Gespräch nur distant dasitzt oder nicht.

Nicht nur die Haltung, sondern auch die räumliche Distanz bestimmt das Gesamtbild der nonverbalen Kommunikation. Beispiele dafür wie Körpersprache 
falsch eingesetzt wird und keine erfolgreiche Rede ermöglicht, auch wenn deren Inhalt noch so gut sein mag, zeigen einem, dass nur die treffenden Worte nicht genügen, damit die Rede gut ankommt. Die treffende Körperhaltung ist sehr wichtig beim Gelingen der Kommunikation. Man stelle sich einen Redner vor, der gebeugt, langsam sich zum Rednerpult schleppt, dessen Blick auf das Präsentationsblatt geheftet bleibt, wenn er liest oder, wenn er dieses zu einer Rolle zusammendreht und es dann wie einen Knüppel in die Hände hält, später dann mit den Füßen auf- und abfedernd wartet, dass er angekündigt wird und beginnen kann zu reden, während der Rede heftig gegen das Mikrofon klopft, wenn man ihm sagt, er spreche zu leise, er konzentriert sich nur auf sein Manuskript, welches er glatt zu streichen versucht und schaut nur kurz verstohlen zum Publikum auf, dann verschränkt er seine Arme vor der Brust, weil sie dort nicht so stark zittern, die Hände gibt er frei nur wenn er umblättern muss und dann gerät er ins Stocken und überspielt die Redepausen durch ein ständiges Räuspern. Dieses Beispiel zeigt, dass in einem Gestus sich Gemütsfassungen, Handlungsintentionen widerspiegeln. Jeder Mensch, der an der Kommunikation beteiligt ist, ist in der Sprechaktion einem Wechselverhältnis aus Einflüssen der äußeren und inneren Situationen ausgesetzt. Er muss Einstellungen und Erwartungen der Hörer antizipieren, um dementsprechend die Gestaltung seines Sprechens zu modellieren (vgl. Jones, 2018). Verhaltensformen wie z.B. den Kopf hängen lassen, mit der Faust auf den Tisch schlagen, sich in die Brust werfen, die Fäuste ballen, die Zähne zusammenbeißen, die Stirn in Falten ziehen, das Gesicht in den Händen vergraben, zeigen, dass die äußere Haltung viel mit der inneren Haltung zu tun hat.

Beispiele dafür gibt es nicht nur im Alltag, sondern auch in literarischen Texten. Die Ausdrucksmerkmale des Körpers, der Gang, die Handbewegungen schließen auf die Wesensart eines Menschen. Ein sprechendes Beispiel dafür ist die Erzählung „Meister Pfriem“ von den Brüdern Grimm.

Ging er auf die Straße, so ruderte er heftig mit beiden Armen und einmal schlug er einem Mädchen, das Wasser trug, den Eimer hoch in die Luft, daß er selbst davon begossen ward [...] Er war seines Handwerks Schuster, und wenn er arbeitete, so fuhr er mit dem Draht so gewaltig an, daß er jedem, der sich nicht weit genug hielt, die Faust in den Leib stieß. [...] Er blieb keine Viertelstunde ruhig sitzen. (Brüder Grimm, 1843)

Ein anderes Beispiel für gestisches Ausdrücken ist in der Erzählung „Der Tänzer“ von Robert Walser zu finden.

Er verspottete den Boden mit seinen Beinen, so wenig Schwere kannte er, und so leicht schritt er dahin. Eine graziöse Musik spielte zu seinem Tanz. [...] Wie ein Engel flog er durch die Luft... Es war als liebe die Luft Ihren Liebling, den göttlichen Tänzer. Wenn er aus der Luft niederschwebte, so war es weniger ein Fallen, als ein Fliegen, ähnlich wie ein großer Vogel fliegt, der nicht fallen kann, 
und wenn er den Boden wieder mit seinen leichten Füßen berührte, so setzte er auch sogleich wieder zu neuen kühnen Schritten und Sprüngen an, als sei es ihm unmöglich mit dem Tanzen und Schweben aufzuhören. [...] indem er tanzte, machte er den schönsten Eindruck, den ein junger Tänzer zu machen vermag, nämlich den, daß er glücklich sei im Tanze. (Walser, 1914)

Ein weiteres Beispiel, in dem man verbale und nonverbale Kommunikation, gestisches Sprechverhalten erkennt, ist in Thomas Manns Erzählung „Mario und der Zauberer" wiedergegeben. In Thomas Manns Erzählung sind verbale und nonverbale Kommunikation zu einer unauflöslichen Einheit verbunden. Gestisches Sprechverhalten, Aspekte des Redeflusses, Pausen, Verlegenheitslaute, Bewegungsverhalten, Lautheit der Stimme verdeutlichen das. Wie die Gestalten sich zu Cipolla sprachlich und gestisch folgsam verhalten, wie Befehlen und Gehorchen in den Kunststücken des Hypnotiseurs in einer unaufhörlichen Folge grotesker Versuche das Publikum zu beherrschen, werden in der Erzählung beschrieben. Das soll im Folgenden am Beispiel einiger Textausschnitte gezeigt werden: „Man mußte zugeben, daß er seine Worte nicht besser hätte wählen können, um die Wasser zu trüben und seelische Verwirrung anzurichten. Der Widerspenstige zögerte nervös bevor er zugriff. [...] wenn auch rednerisch immer noch die Umschreibung herrschte“ (Mann, 1988: 291), bekam das Publikum

[...] in einer langwierigen Serie komischer, aufregender, erstaunlicher Versuche [...] vom Unscheinbaren bis zum Ungeheuerlichen alles zu sehen [...] und den grotesken Einzelheiten folgte ein lachendes, kopfschüttelndes, sich aufs Knie schlagendes, applaudierendes Publikum, das deutlich im Bann einer Persönlichkeit von strenger Selbstsicherheit stand. [...] Cipolla winkte Mario zu, indem er die Hand vor die Nase hielt und abwechselnd den Zeigefinger lang aufrichtete und zum Haken krümmte. Mario gehorchte. [...] Der Zauberer sprach zu Mario klar akzentuiert, wenn auch in den Tonfall seiner Worte etwas Sattes und Paschahaftes, etwas von Ränkelei und Übermut eingetreten war. [...] Es war greulich, wie der Betrüger sich lieblich machte, die schiefen Schultern kokett verdrehte, die Beutelaugen schmachteln ließ und in süßlichem Lächeln seine splittrigen Zähne zeigte. [...] Cipolla war mit einem Satz vom Stuhle aufgesprungen. Er stand da mit abwehrend seitwärts gestreckten Armen, als wollte er rufen: ,Halt! Still! Alles weg von mir! Was ist das?' (Mann, 1988: 293-310)

Alles führte in dem Verhalten der Titelgestalten zu dem „Ende mit Schrecken, ein höchst fatales Ende. Und ein befreiendes Ende dennoch“". (Mann, 1988: 310)

Der Grimmsche Text zeigt, wie man im täglichen Leben ständig Handlungen und Verhaltensweisen anderer Menschen gegenübergestellt wird. Man gelangt so zu der Erkenntnis, dass das Handeln und der Charakter eines Menschen zusammenhängen. Daraus erwächst das Bedürfnis, Handlungsmotive zu erfassen, sie aus den sachlichen Zusammenhängen oder aus den Eigenschaften der Urheber 
zu verstehen. Man gelangt schließlich zu einer Beurteilung von Handlungs- und Verhaltensweisen der Menschen. So kann man einen Menschen aus seinem Verhalten charakterisieren, aus seiner Sprechweise, Lautstärke, Wortwahl, aus seinem ganzen Körperverhalten. Es ist nicht unbedingt notwendig, dass nur das Äußere, das Aussehen in der Charakterisierung eine Rolle spielt. Gefühle und Gedanken widerspiegeln sich zwar auch im abstoßenden Aussehen des Meisters, aber viel schlimmer ist sein Verhalten. Von äußeren, greifbaren Aspekten wird auf das Wesentliche, auf das Innere, auf das Denken und Fühlen des Meisters geschlossen. Sein grobesVerhalten, seine brutalen Bewegungen schließen auf seine Menschenfeindlichkeit.

Die beiden anderen Textbeispiele stellen unterschiedliche Aspekte der von Menschen ausgeübten Kunst dar. Es geht einerseits um die wahre Kunst, die den Menschen innerlich bereichert. Der Tänzer bringt mit seiner Tanzkunst Erhabenheit, Schönheit, Freiheit. Seine leichten schwebenden Bewegungen lassen den Zuschauer bzw. den Leser dieser Zeilen mitschwingen. Seine Körperhaltung und sein Talent üben eine positive Wirkung auf den Zuschauer aus. Die Schönheit seiner Tanzkunst ist erhebend. Grazie und Größe vereinen sich in seinen Körperbewegungen. Diese drücken vielmehr aus als Worte sagen können. Gäbe es nicht den wahren Künstler, den Tänzer, hätte es nicht andere Leute seinesgleichen gegeben, die das Echte und Schöne schätzen. Sieht man seine Körperbewegungen, so sieht man sich selbst, man könnte fliegen, immer weiter, immer höher fliegen und von oben die Welt sehen. Der Tänzer bewegt sich mit Leichtigkeit und Eleganz auf der Bühne, wo er in seinem Element ist. Seine Körpersprache sagt alles, was man über seine Kunst sagen kann.

Der Zauberer in der Erzählung von Thomas Mann zeigt die dunkelsten Seiten einer Zauberkunst, die eigentlich keine Kunst ist, sondern nur die grausamsten Stufen der Angst, der das Publikum machtlos ausgesetzt ist. Durch sein verbales, nonverbales und paraverbales Verhalten tyrannisiert der Zauberer das Publikum, er reißt es an sich und lässt es blindlings das tun, was er will. Das Publikum wird zum Objekt, zum Opfer des Zauberkünstlers, der durchgreift, zupackt, erniedrigt und beherrscht, während er auf seine grausame Nüchternheit pocht.

\section{Körpersprachliche Signale}

Beim Sprechen, bei einem Gespräch von Angesicht zu Angesicht gestikulieren Menschen oft. Diese Gestik gehört zu den Äußerungsformen, die unbewusst das Sprechen begleiten. Sie sind körperliche Signale, die die Gesprächsführung mitsteuern, sie fügen der Rede keine Informationen hinzu, sondern sie erleichtern den Sprechprozess. Die Gesten gehen der sprachlichen Äußerung voraus, sie treten vor dem Wort auf, weil die Konzeptualisierung auf das Signal der Gesteplanung wartet (vgl. Papst-Weinschenk, 2004: 54). 
Bei den Gesten handelt es sich nicht um gestikulierendes Sprechen, nicht in dem Fall, wenn das Gestikulieren ausschließlich illustrativen, motorischen Charakter hat. Gesten haben als körperliches Signal einer inneren Haltung und eines Handlungsmotivs eine wichtige Funktion. Gesten zeigen sich in Handlungsintentionen. Sie können auch als eine stärkere Artikulation bestimmter Laute oder Wortgruppen auftreten z.B. bei den Wahlreden der Politiker. In diesem Fall sind auch die Kontextbedingungen ausschlaggebend nicht nur der Auftritt des Redners, denn jede Rede steht in einem Kontext von Erwartungen und Voraussetzungen, die die Bedingungen des Erstellens und des Vortragens der Rede durch den Redner und der Rezeption durch das Publikum ausmachen. Diese Bedingungen sind situativer und normativer Natur. So erfordert die Situation einer Wahlrede, klare Darlegungen der Ziele, Appellen zur Wahlentscheidung, die nicht nur durch eine ausdrucksstarke Artikulation erreicht werden kann, sondern der ganze Kommunikationsvorgang muss gut durchdacht werden. Wichtig ist auch die Weitergabe von Redeinhalten durch sogenannte Meinungsbildner. Eine Rede ist dann wirksam, wenn sie nicht nur sprachlich gut aufgebaut ist und rhetorische, stilistische Mittel aufweist, sondern auch angemessen vorgetragen wird. Heutzutage nutzen Politiker, und nicht nur sie, die Plattform Talkshow, um an die Emotionen des Publikums zu appellieren. Die kommunikative Grundfunktion der Talkshows ist informativ-persuasiv d.h. sie haben neben ihrer Unterhaltungsfunktion vor allem die Funktion politisches Handeln zu analysieren, $\mathrm{zu}$ kritisieren, $\mathrm{zu}$ rechtfertigen im Rahmen spezifischer kommunikativer Bedingungen, die die verbale und gleichzeitig auch die nonverbale Kommunikation steuern und beeinflussen (vgl. Girnth, 2002).

Körpersignale tragen in hohem Maße dazu bei, durch bestimmte Gesten die verbale Kommunikation zu bekräftigen. Das sieht man bei den Politikern darin, dass sie oft wichtige Themen, Aspekte, Argumente in ihren Reden mit ihrer zur Faust geballten rechten/ oder linken Hand, der sogenannten Aktionshand, durch pulsierende Schläge demonstrativ unterstützen. Es gibt ganz unterschiedliche Facetten in der nonverbalen Kommunikation, die unterschiedlich gedeutet werden können.

Es gibt eine enge Korrelation zwischen der verbalen und der nonvokalen Kommunikation, wenn z.B. jemand vor dem Gesprächspartner die Augenbrauen hochzieht, kann er als Reaktion eine weitere Erklärung bekommen, um die Skepsis zu beheben. Bei den körperlichen Signalen geht es um die Frage, ob sich in diesen Ausdrucksformen des Körpers Merkmale auffinden lassen, die für den Sprachkodes typisch sind. Nach semiotischen Methoden wird das Sprechen mit Gesten mit Methoden der Sprachwissenschaft analysiert, indem die Kineme, als die kleinsten bedeutungstragenden Bewegungseinheiten, analog zu den Monemen bzw. Morphemen analysiert werden (vgl. Papst-Weinschenk, 2004: 50). 


\section{Fazit}

Es gibt einen Zusammenhang zwischen der inneren und der äußeren Haltung innerhalb der Kommunikation. Das heißt, dass die psychische und physische Verfassung der Kommunikationspartner während des Sprechens und des Hörens Hand in Hand gehen. Gefühle kommen in der Körpersprache zum Ausdruck und durch körperliche Veränderungen verändert sich auch die Gefühlslage der Kommunikationsteilnehmer. Auf einen guten Blickkontakt, auf einen festen Stand und auf einer offenen Haltung muss man vor einem Zuhörerkreis achten. In der Kommunikation geht es um Handeln mit und ohne Sprache, um Intentionen, um die Umstände und Beziehungen zwischen den Menschen, um Haltungen hinter den Worten, um körperliches Verhalten im Allgemeinen, welches innere Haltungen zum Ausdruck bringt.

\section{Bibliografie/ Literatur}

Dietrich, R. 2002. Psycholinguistik, Stuttgart: Metzler.

Girnth, H. 2002. Sprache und Sprachverwendung in der Politik. Eine Einführung in die linguistische Analyse öffentlich-politischer Kommunikation, Tübingen: Niemeyer.

Brüder Grimm. 1843. "Meister Pfriem", in Bock, I.H. (Hrsg.) 1974. Sprachlehre, Bucuresti: EDP: 129.

Helfrich, H. und H.G. Wallbott. 1980. 'Theorie der nonverbalen Kommunikation. Kommunikatives Handeln', in Althaus, H.P., Henne, H. und H.E. Wiegand. 1980. Lexikon der Germanistischen Linguistik. Kommunikatives Handeln. Band 3, Tübingen: Niemeyer: 267-275.

Hübler, A. 2001. Das Konzept „Körper" in den Sprach- und Kommunikationswissenschaften, Tübingen- Basel: Francke.

Hübler, A. 2007. The Nonverbal Shift in Early Modern English Conversation, Amsterdam/Philadelphia: John Benjamins Publishing Company.

Jones, Ph. M. 2018. Exactly What to Say: The Magic Words for Influence and Impact, Vancouver, B.C.: Page Two Books.

Keller, R. 1994. Sprachwandel: Von der unsichtbaren Hand in der Sprache, Tübingen: Francke.

Mann, T. 1988. Erzählungen, Leipzig: Philipp Reclam jun.

Navarro, J. 2010. Menschen lesen. Ein FBI-Agent erklärt, wie man Körpersprache entschlüsselt Mitarbeit: Karlins, Marvin, München: mvgverlag.

Navarro, J. 2018. The Dictionary of Body Language, New York: Thorsons Publishing Group/Harper Collins.

Nörth, W. 1985. Handbuch der Semiotik, Stuttgart: Metzler.

Papst-Weinschenk, M. 2004. Grundlagen der Sprechwissenschaft und Sprecherziehung, München und Basel: Reinhard. 
Schmidt, V. 2019. Mit den Ohren sehen. Die Methode des gestischen Sprechens an der Hochschule für Schauspielkunst Ernst Busch Berlin, Berlin: Theater der Zeit Verlag.

Stadler, B. 1978. Sprechhandeln und Grammatik. Band 2: Sprechhandlungsorientierte Grammatik, München: Oldenbourg.

Walser, R. 1914. "Der Tänzer", in von Wiese, B. (Hrsg.) 1978. Deutschland erzählt, Frankfurt am Main: Fischer: 61-62.

Watzlawick, P. 1974. "Axiome der Kommunikation", in Heringer, H.J. 2004. Interkulturelle Kommunikation, Tübingen und Basel: Francke: 18-22.

\section{The author}

Mihaela Parpalea is an Associate Professor at Transilvania University of Braşov where she teaches Contemporary German Language (CGL) courses from the level of first year, up to MA level. Her primary research interest consists in studying and teaching general linguistics. The author has published several professional reviews in nationally known methodical journals (Revue roumaine de linguistique LIX, (1) 2014), as well as other scientific papers in Romanian and international well-known professional journals: Sprachmuster und Sozialstruktur, Nachdenken über Sprache unter kommunikativfunktionalem Aspek, Sprachliche Aspekte des Schweigens in verschiedenen Kulturen, Verkaufsgesprächstypen-Ein Thema für die Linguistik, Kultur- und kontextgebundenes Kommunikationsverhalten-Sagen, meinen und verstehen, Formen des Sprachgebrauchs innerhalb der Beziehung Sprecher-Sprache, Wie man durch bestimmte Sprachformen zeitgemäß sein kann. 